\title{
Migraine is associated with an increased risk for benign paroxysmal positional vertigo: a nationwide population-based study
}

\author{
Chia-Huei Chu ${ }^{1,6}$, Chia-Jen Liü ${ }^{2,7}$, Liang-Yu Lin ${ }^{3,8}$, Tzeng-Ji Chen ${ }^{4}$ and Shuu-Jiun Wang $5,9,10,11^{*}$
}

\begin{abstract}
Background: There is evidence suggesting that migraine may be associated with vertigo. The aim of this study was to assess the risk of benign paroxysmal positional vertigo (BPPV), the most common form of vertigo, in patients with migraine using a population-based dataset.

Methods: The National Health Insurance Research Database in Taiwan was searched for migraine patients and was also used to select an age- and sex-matched cohort of subjects without migraine. The analyses included 8266 migraine patients and 8266 controls. The incidence rates of BPPV in the two cohorts were compared. Cox proportional hazard models were used to identify risk factors for BPPV in migraine patients.

Results: In the migraine cohort, $1.11 \%$ of the patients developed BPPV compared to $0.5 \%$ of the controls. The incidence rate ratio was 2.03 ( $95 \% \mathrm{Cl} 1.41-2.97 ; \mathrm{p}<0.001$ ). Cox proportional hazards analysis showed that age $\geq 40$ years (HR 2.20; $95 \% \mathrm{Cl} 1.40-3.45 ; p=0.001$ ), coronary artery disease ( $\mathrm{HR} \mathrm{4.62;} 95 \% \mathrm{Cl} 1.12-19.01 ; p=0.034$ ), and the number of outpatient department visits to neurologists because of migraine (HR 2.93; $95 \% \mathrm{Cl} 2.50-3.44 ; \mathrm{p}>0.001$ ) were associated with an increased risk for BPPV.

Conclusion: The results showed that patients with migraine had a 2.03-fold increased risk of developing BPPV compared with age- and sex-matched controls. Although BPPV may not be a common condition in migraine patients, migraine sufferers with vestibular symptoms should alert physicians to the possibility of BPPV, particularly if patients are aged $\geq 40$ years, have a history of coronary artery disease, or have frequent visits to neurologists clinics because of migraine.
\end{abstract}

Keywords: Migraine; Benign paroxysmal positional vertigo; Risk factors; Incidence rate

\section{Background}

Both migraine and vertigo rank among the most common complaints in the general population. Making an association between migraine and dizziness dates back to the 19th century when Liveing first mentioned their connection [1]. Later, a Kayan and Hood reported that migraine patients had a much higher incidence of vertigo than the tension headache patients $(26.5 \%$ versus $7.8 \%$ ) [2]. An epidemiological study had reported that there might be a link between migraine and vertigo

\footnotetext{
*Correspondence: sjwang@vghtpe.gov.tw

${ }^{5}$ Department of Neurology, Neurological Institute, Taipei Veterans General

Hospital, Taipei, Taiwan

${ }^{9}$ Department of Neurology, National Yang-Ming University School of

Medicine, Taipei, Taiwan

Full list of author information is available at the end of the article
}

[3]. In Taiwan, migraine has a prevalence ranging from $8 \%$ to $15 \%$ [4]. People between the ages of 25 and 55, usually the most productive years of life, have the highest prevalence of migraine [5].

A number of studies have reported findings which suggesting that repeated migraine attacks can result in progressive structural and functional alterations of the brain [6]. Migraine also is a risk factor for ischemic stroke and there are reports of ischemic stroke occurring during migraine attacks $[6,7]$.

The prevalence of dizziness and vertigo in the general population is approximately $20 \%$ to $30 \%$ [3]. Many migraine patients suffered from dizziness or vertigo. Benign paroxysmal positional vertigo (BPPV) is the most frequent cause of vertigo [8]. It can be easily diagnosed and usually rapidly corrected. Studies have reported that 
the prevalence ranges from 10.7 to 64.0 per 100,000 population [8] with a life time prevalence of $2.4 \%$. The prevalence is increased among elderly persons and among women, with peak onset between 50 and 60 years of age and a female-to-male ratio of 2:1 to 3:1 [9]. BPPV is characterized by brief spinning sensations lasting less than $1 \mathrm{~min}$, which are generally induced by an abrupt change in head position with respect to gravity. It is believed to be caused by otolith, i.e., small calcium carbonate crystals that enter the semicircular canals from the utricle, one of the otolith organs in the inner ear. A change of the head position results in these crystals travelling within the semicircular canals which drives movement of the endolymph causing the sensation of vertigo [8]. Clinically, BPPV can usually be diagnosed based on the nystagmus induced by Dix-Hallpike maneuver $[8,10]$ and can often be successfully treated immediately using Epley's canalith-repositioning maneuver [8].

Previous researches have shown that migraine and vestibular dysfunction may have potential interactions and relationships via several conditions including BPPV or other disorders related to inner ear dysfunction $[11,12]$. Therefore, the aim of this study was to explore the risk of BPPV in migraine patients.

\section{Materials and methods}

\section{Data sources}

Taiwan launched the National Health Insurance (NHI) program in 1995 to finance health care for all citizens. The NHI system now covers $99.9 \%$ of Taiwan's population, and $93 \%$ of the country's hospitals and clinics are NHI-contracted [13]. The NHI is a mandatory, singlepayer social insurance system, offering almost universal medical care. It includes coverage for outpatient, inpatient, emergency, dental, traditional Chinese medicine services, and prescription drugs. In 1999, the Bureau of National Health Insurance made patient data available electronically through the National Health Insurance Research Database (NHIRD) project. The multiple NHI databases (i.e. NHI enrollment files, claims data, and registry of drug prescriptions) provide comprehensive utilization and enrollment information for all patients under the NHI program. This study used NHI databases that are managed and publicly released by the National Health Research Institute of Taiwan. The confidentiality of the data abides by the regulations of the Bureau of the NHI and the National Health Research Institute. All information that would potentially expose a specific individual to be identified has been encrypted. Since the NHI dataset consists of de-identified secondary data for research purposes, this study was exempt from full review by the institutional review board.

\section{Study population}

A retrospective cohort study was conducted using a dataset from January 1, 2000 to December 31, 2009. Patients who were diagnosed with migraine by a neurologist according to the migraine diagnosis codes $(346.0 \times, 346.1 \times$, and $346.9 \times)$ in the International Classification of Diseases, Ninth Revision, Clinical Modification (ICD-9-CM) were enrolled. Patients diagnosed with migraine at an age $<20$ or $\geq 65$ years were excluded. Patients with antecedent BPPV (386.11) within one year before the diagnosis of migraine were also excluded. In addition, those diagnosed with acoustic neuroma (225.1), Meniere's disease (386.0×), vestibular neuritis (386.12), labyrinthitis $(386.3 \times)$, sudden hearing loss (388.2), or head injury (310.2, 800.x-804.x, 850.x-854.x, 870.x-873.x, 907.0, 907.1, 959.0x, and V15.52) at any time within the study period were also excluded from the analysis. Subjects without migraine were randomly selected from the same database for the matched (non-exposure) cohort. Each migraine patient was matched with one non-exposure subject by age and sex within the same observational period.

Both the migraine cohort $(n=8,266)$ and the matched cohort $(n=8,266)$ were followed up until the development of BPPV, transfer out of the NHI program, death, or the end of the year 2010 .

\section{Outcome}

The outcome variable of this study was new occurrence of BPPV, as defined by a compatible ICD-9-CM code 386.11 that was coded by a neurologist or an otorhinolaryngologist.

\section{Statistical analysis}

Categorical variables were presented as counts and percentages, and the chi-square test was performed for group comparisons. The incidence rates (per 100,000 person-years) with $95 \%$ confidence intervals (CIs) and incidence rate ratios (IRRs) of BPPV in both groups were analyzed. The Kaplan-Meier method and the Cox proportional hazard models were used to explore the associations between each possible confounding factor and BPPV. Factors with $p<0.1$ in univariate analyses were then included in a multivariable model. Extraction and computation of data were performed with the Perl programming language (version 5.12.2). A Microsoft SQL Server 2005 (Microsoft Corp., Redmond, WA, USA) was used for data linkage, processing, and sampling. The statistical analyses were carried out with IBM SPSS statistical software version 19.0 for Windows (IBM Corp., Armonk, New York, USA). Statistical significance was defined as a $p$ value less than 0.05 . 


\section{Results}

Demographic characteristics of the study population

During the 10-year study period, a total of $14,164 \mathrm{mi}-$ graine patients were diagnosed or had diagnoses confirmed by neurologists. After excluding patients with aged $<20(n=1,255)$ or $\geq 65$ years $(n=1,304)$, BPPV before migraine diagnosis $(n=65)$, acoustic neuroma $(n=21)$, Meniere's disease $(n=789)$, vestibular neuritis $(n=372)$, labyrinthitis $(n=38)$, sudden hearing loss $(n=35)$, and head injury $(n=2,019)$ diagnosed at any time within the study period, the final sample consisted of 8266 patients for the analyses.

The demographic characteristics of the migraine cohort and matched cohort are presented in Table 1. In each cohort, the median age was 38 years and $72 \%$ of the cohort were females. Among the migraine patients, $8.93 \%$ were diagnosed as having migraine with aura. No significant differences were found between the migraine cohort and matched cohort in comorbidities (Table 1). The median follow-up duration was 6.24 years for the matched cohort and 6.29 years for the migraine cohort.

Table 1 Baseline characteristics of patients with migraine and matched cohort

\begin{tabular}{|c|c|c|c|c|c|}
\hline \multirow[t]{2}{*}{ Characteristics } & \multicolumn{2}{|c|}{$\begin{array}{l}\text { Migraine cohort } \\
n=8266\end{array}$} & \multicolumn{2}{|c|}{$\begin{array}{l}\text { Matched cohort } \\
n=8266\end{array}$} & \multirow[t]{2}{*}{$P$ value } \\
\hline & No. & $\%$ & No. & $\%$ & \\
\hline $\begin{array}{l}\text { Median age, years } \\
\text { (interquartile range) }\end{array}$ & $38(29-48)$ & & $38(29-48)$ & & \\
\hline \multicolumn{6}{|l|}{ Age, years } \\
\hline$<40$ & 4408 & 53.3 & 4408 & 53.3 & 1.000 \\
\hline$\geq 40$ & 3858 & 46.7 & 3858 & 46.7 & \\
\hline \multicolumn{6}{|l|}{ Sex } \\
\hline Female & 5952 & 72.0 & 5952 & 72.0 & 1.000 \\
\hline Male & 2314 & 28.0 & 2314 & 28.0 & \\
\hline \multicolumn{6}{|l|}{ Comorbidities } \\
\hline Diabetes mellitus & 811 & 9.8 & 810 & 9.8 & 0.979 \\
\hline Hypertension & 1460 & 17.7 & 1460 & 17.7 & 1.000 \\
\hline Heart failure & 159 & 1.9 & 152 & 1.8 & 0.689 \\
\hline COPD & 1081 & 13.1 & 1079 & 13.1 & 0.963 \\
\hline Asthma & 873 & 10.6 & 870 & 10.5 & 0.939 \\
\hline Chronic kidney disease & 541 & 6.5 & 539 & 6.5 & 0.950 \\
\hline Coronary artery disease & 35 & 0.4 & 29 & 0.4 & 0.452 \\
\hline Dyslipidemia & 1429 & 17.3 & 1429 & 17.3 & 1.000 \\
\hline Cirrhosis & 67 & 0.8 & 58 & 0.7 & 0.419 \\
\hline Autoimmune diseases & 588 & 7.1 & 582 & 7.0 & 0.856 \\
\hline Cerebrovascular disease & 876 & 10.6 & 869 & 10.5 & 0.859 \\
\hline Previous BPPV & 69 & 0.8 & 58 & 0.7 & 0.327 \\
\hline Migraine with aura & 738 & 8.93 & & & \\
\hline
\end{tabular}

Abbreviations: COPD chronic obstructive pulmonary disease, BPPV benign paroxysmal positional vertigo

\section{Comparisons of the incidence rates of BPPV between migraine patients and the matched cohort}

Comparisons of BPPV incidence rates between the migraine patients and the matched control group are presented in Table 2.

Ninety-two $(1.11 \%)$ patients in the migraine cohort and $45(0.5 \%)$ subjects in the matched cohort developed BPPV (Fisher's exact test, $p<0.001$ ). The migraine cohort had a greater risk of developing BPPV than the matched cohort (IRR $=2.03$; $95 \%$ CI $1.41-$ 2.97, $\mathrm{p}<0.001$ ). Both the older (aged $\geq 40$ years) and the younger (age $<40$ years) patients with migraine had a higher incidence of BPPV than the matched controls ( 26.8 vs. 14.6 per 100,000 person-years and 10.6 vs. 4.0 per 100,000 person-years, $p=0.004$, respectively). The female migraine subgroup had a higher IRR than the male subgroup, and this reached statistical significance in the female subgroup (19.7 versus 14.1 per 100,000 person-years, Fisher's exact test, $p<0.001)$. Patients diagnosed as having migraine without aura had a significantly higher IRR compared with the matched controls $(\operatorname{IRR}=1.99,95 \%$ CI 1.35-2.97, $p<0.001)$. Only 10 of the 738 patients who had migraine with aura developed BPPV, and no significant difference in IRR was found between the migraine patients and controls $(\mathrm{IRR}=2.48,95 \%$ CI 0.71-10.81, $p=0.123$ ) (Table 2).

The cumulative incidence of BPPV in patients with migraine was significantly higher than that in the matched cohort (log rank, $p<0.001$, Fig. 1).

\section{Risks factors for BPPV in migraine patients}

Univariate Cox regression analysis revealed a significantly increased risk of BPPV in migraine patients with the following characteristics: age $\geq 40$ years, hypertension, chronic obstructive pulmonary disease (COPD), coronary artery disease (CAD), cerebrovascular disease, and number of outpatient department (OPD) visits to see a neurologist for migraine (all $\mathrm{p}<0.05$ ). If the factors in univariate analysis have a $p$-value $\leq 0.1$, they were included in multivariate analysis. The results of multivariable Cox proportional hazards analysis showed there was a significantly higher hazard risk of developing BPPV in the migraine cohort associated with age $\geq 40$ years $(\mathrm{HR}=2.2, p=0.001)$, CAD $(\mathrm{HR}=4.62$, $p=0.034$ ), and the number of visits to neurologist's OPD for migraine (HR $=2.93, p<0.001)$ (Table 3$)$.

\section{Discussion}

This nationwide, population-based, cohort study demonstrated that migraine was associated with an increased risk of developing BPPV that was 2.03 times higher than that of the matched controls. The incidence rate was 18.1 per 100,000 person-years in the migraine cohort in contrast to 8.9 in the matched control cohort. 
Table 2 The risk of benign paroxysmal positional vertigo (BPPV) for patients with migraine and matched cohort

\begin{tabular}{|c|c|c|c|c|c|c|}
\hline \multirow[t]{2}{*}{ Characteristics } & \multicolumn{2}{|c|}{ Patients with migraine, $n=8266$} & \multicolumn{2}{|c|}{ Matched cohort, $n=8266$} & \multirow[t]{2}{*}{ IRR (95 \% CI) } & \multirow[t]{2}{*}{$P$ value } \\
\hline & BPPV No. & Per 10,000 person-year & BPPV No. & Per 10,000 person-year & & \\
\hline Total & 92 & 18.1 & 45 & 8.9 & $2.03(1.41-2.97)$ & $<0.001$ \\
\hline \multicolumn{7}{|l|}{ Age } \\
\hline$<40$ & 29 & 10.6 & 11 & 4.0 & $2.63(1.28-5.84)$ & 0.004 \\
\hline$\geq 40$ & 63 & 26.8 & 34 & 14.6 & $1.84(1.19-2.88)$ & 0.004 \\
\hline \multicolumn{7}{|l|}{ Sex } \\
\hline Male & 20 & 14.1 & 12 & 8.5 & $1.66(0.77-3.72)$ & 0.168 \\
\hline Female & 72 & 19.7 & 33 & 9.1 & $2.17(1.42-3.39)$ & $<0.001$ \\
\hline \multicolumn{7}{|l|}{ Aura } \\
\hline With aura & 10 & 24.2 & 4 & 9.8 & $2.48(0.71-10.81)$ & 0.123 \\
\hline Without aura & 82 & 17.5 & 41 & 8.8 & $1.99(1.35-2.97)$ & $<0.001$ \\
\hline
\end{tabular}

Abbreviations: IRR incidence rate ratio, $\mathrm{Cl}$ confidence interval

Chi-square tests were used for the group comparisons

Furthermore, there was a higher risk of developing BPPV associated with three factors: migraineurs aged $\geq 40$ years $(\mathrm{HR}=2.20), \mathrm{CAD}(\mathrm{HR}=4.62)$ as well as neurologists' OPD visit. To the best of our knowledge, this research is the first population-based study to demonstrate that migraine is associated with an increased risk for BPPV.

Comorbid conditions such as hypertension, coronary artery disease, and cerebrovascular disease would be expected to be more common in a non-migraine population than in a non-migraine population, however, in this study there were no significant differences in prevalence of comorbid conditions between the migraine group and non-migraine control group. The reason for this was that not only were the patients in the migraine group and control group perfectly matched for sex and age but we also tried to match the groups for comorbid conditions (Table 1).

There is now considerable epidemiological evidence linking migraine to vertigo as well as dizziness. Among patients whose are diagnosed as having migraine, about $25 \%$ also complain of having vertigo and about $30 \%$ complain of dizziness [14]. In studies of patients with dizziness, it has been found that such patients have a higher prevalence of migraine than would be expected and in researches of patients with migraine it has been found that such patients have an increased prevalence of vertigo and dizziness [3]. A recent cross-sectional study by Calhoun et al investigated the point prevalence of dizziness or vertigo in patients with migraine [15]. They found that patients who had migraine with aura had a

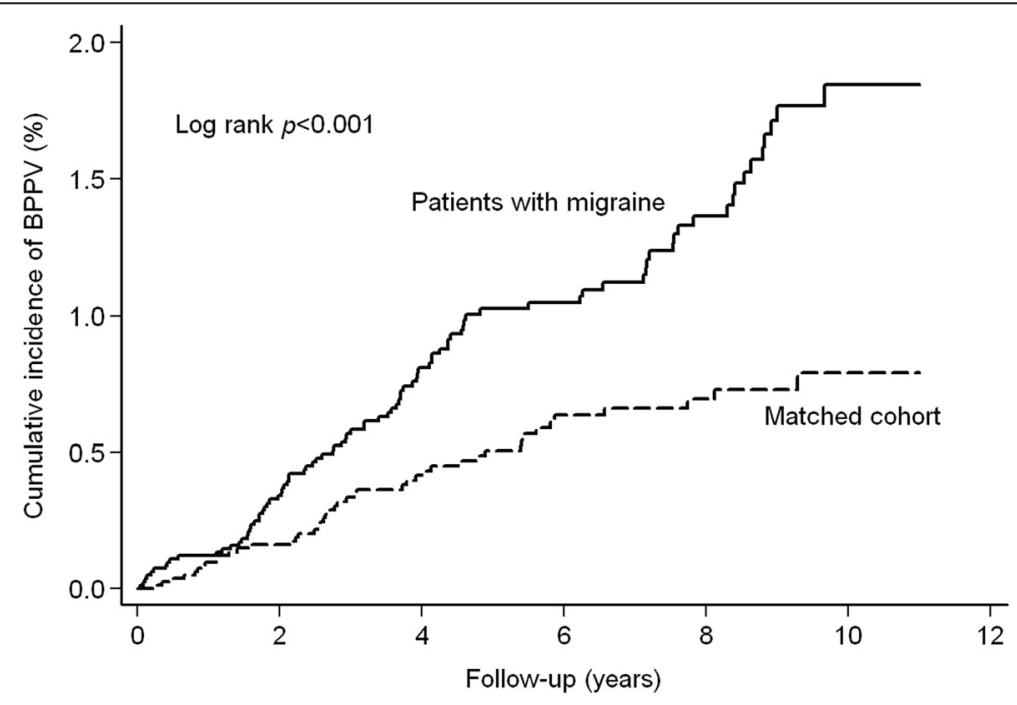

Fig. 1 Cumulative incidence of benign paroxysmal positional vertigo in migraine and matched cohort 
Table 3 Risk factors for benign paroxysmal positional vertigo in patients with migraine

\begin{tabular}{|c|c|c|c|c|}
\hline \multirow[b]{2}{*}{ Variables } & \multicolumn{2}{|c|}{ Univariate analysis } & \multicolumn{2}{|c|}{ Multivariable analysis $^{a}$} \\
\hline & $\mathrm{HR}(95 \% \mathrm{Cl})$ & $P$ value & $\mathrm{HR}(95 \% \mathrm{Cl})$ & $P$ value \\
\hline Age $\geq 40$ years & $\begin{array}{l}2.53 \\
(1.63-3.92)\end{array}$ & $<0.001$ & $\begin{array}{l}2.20 \\
(1.40-3.45)\end{array}$ & 0.001 \\
\hline Male sex & $\begin{array}{l}0.72 \\
(0.44-1.17)\end{array}$ & 0.186 & & \\
\hline \multicolumn{5}{|l|}{ Comorbidities } \\
\hline Diabetes mellitus & $\begin{array}{l}0.58 \\
(0.24-1.42)\end{array}$ & 0.233 & & \\
\hline Hypertension & $\begin{array}{l}1.97 \\
(1.25-3.1)\end{array}$ & 0.003 & & \\
\hline Heart failure & $\begin{array}{l}2.00 \\
(0.63-6.32)\end{array}$ & 0.238 & & \\
\hline COPD & $\begin{array}{l}1.93 \\
(1.16-3.20)\end{array}$ & 0.011 & & \\
\hline Asthma & $\begin{array}{l}1.34 \\
(0.71-2.51)\end{array}$ & 0.366 & & \\
\hline $\begin{array}{l}\text { Chronic } \\
\text { kidney disease }\end{array}$ & $\begin{array}{l}1.33 \\
(0.62-2.88)\end{array}$ & 0.467 & & \\
\hline $\begin{array}{l}\text { Coronary } \\
\text { artery disease }\end{array}$ & $\begin{array}{l}6.35 \\
(1.56-25.8)\end{array}$ & 0.010 & $\begin{array}{l}4.62 \\
(1.12-19.01)\end{array}$ & 0.034 \\
\hline Dyslipidemia & $\begin{array}{l}1.56 \\
(0.95-2.56)\end{array}$ & 0.080 & & \\
\hline Cirrhosis & $\begin{array}{l}2.78 \\
(0.69-11.3)\end{array}$ & 0.152 & & \\
\hline Autoimmune diseases & $\begin{array}{l}1.64 \\
(0.82-3.27)\end{array}$ & 0.159 & & \\
\hline $\begin{array}{l}\text { Cerebrovascular } \\
\text { disease }\end{array}$ & $\begin{array}{l}2.60 \\
(1.60-4.22)\end{array}$ & $<0.001$ & & \\
\hline Previous BPPV & $\begin{array}{l}3.54 \\
(0.87-14.39)\end{array}$ & 0.077 & & \\
\hline $\begin{array}{l}\text { Neurologists' OPD visit } \\
\text { for migraine }\end{array}$ & $\begin{array}{l}3.00 \\
(2.56-3.51)\end{array}$ & $<0.001$ & $\begin{array}{l}2.93 \\
(2.50-3.44)\end{array}$ & $<0.001$ \\
\hline Migraine with aura & $\begin{array}{l}1.38 \\
(0.71-2.65)\end{array}$ & 0.340 & & \\
\hline
\end{tabular}

Abbreviations: $H R$ hazard ratio, $\mathrm{Cl}$ confidence interval COPD chronic obstructive pulmonary disease, BPPV benign paroxysmal positional vertigo

${ }^{\mathrm{a} A l l}$ factors with $p<0.1$ in univariate analyses were selected for Cox multivariable analysis

The reference groups for each variable were as follows: Age $<40$ years, female, without co-morbidities, without neurologist OPD visit, or without aura, respectively

prevalence of dizziness or vertigo that was twice as high (24.5\% versus $12.1 \%)$ as patients with migraine without aura and that the prevalence of dizziness or vertigo was significantly associated increased age. They also reported a significant relationship between severity of migraine pain and increased complaints of vertigo. Almost half of the migraineurs reported concomitant dizziness or vertigo when migraine pain was present at an intensity of 7 or greater (on a scale of 1-10). In our study, age $\geq 40$ years was noted to be a risk factor for
BPPV in patients with migraine, and this finding was also presented in our study. Warninghoff et al carried out the first study to investigate systematically the comorbidities of vertiginous diseases [16]. In this survey study of 131 participants with various kinds of vertiginous diseases, no increased prevalence $(9.4 \%)$ of $\mathrm{mi}$ graine was found compared with an epidemiological study of a 6-month prevalence $11.2 \%$ for migraine. The relatively small sample size may explain the finding. A retrospective study of 476 patients with BPPV conducted by Uneri revealed that migraine and motion sickness were three times more common in patients with BPPV than in the general population and a family history of migraine $(58.4 \%)$ was also more common [17]. Another research by Fararilli et al investigated 186 BPPV in patients with migraine [18]. They compared a group of patients with BPPV and migraine with a group of patients with BPPV but without migraine or other type of headache. They found that mean age of BPPV onset was earlier in the former group (39 versus 53 years) and highly recurrent BPPV occurred more often in the former group (19.4\% versus $7.3 \%)$. The findings suggested that the clinical features of BPPV are affected by migraine.

The major strength of this study was its population-based design, which examined a representative cohort of 1 million citizens covered under the NHI in Taiwan. The large sample size and long observation period offered enough power to delineate the differences between the two study groups. To achieve a high validity, the enrolled migraine patients had to be diagnosed only by neurologists, and the outcome variable, new onset BPPV, had to be coded by a neurologist or an otorhinolaryngologist. Additionally, patients with certain common inner ear diseases such as Meniere's disease, acoustic neuroma, vestibular neuritis, labyrinthitis, and sudden hearing loss, and those who had head injury at any time within the study period were excluded since BPPV might be related or secondary to these disorders. The strict inclusion and exclusion criteria minimized possible coding deviations in this claims dataset.

No direct pathophysiological link between migraine and BPPV has yet been established. Baloh in 1997 had postulated that neuro-otological symptoms in migraine patients may originate from vasospasm and/or a certain ion channel disorder [1]. Ishiyama et al once hypothesized that patients with migraine suffered recurrent damage to the inner ear because of vasospasm or some other mechanism, which predisposes them to episodes of BPPV $[19,20]$. Therefore it could be inferred that repetitive vasospasms or disturbance of vestibule-cochlear microvasculature might play a role in inner ear insult, resulting in damages of the epithelium in the vestibule, causing dislodge of the otoconia from utricular macula into semicircular canal, and thus give rise to BPPV. 
The same postulation may also explain the correlation between migraine and various hearing problems that had been largely reported [21, 22]. It should also be noted that a neurogenic inflammation in the inner ear in experimental migraine animal models has been reported [23]. Such recurrent neurogenic inflammation might accelerate degeneration of the otoliths organ. However it remains unclear what triggers this inflammation.

Various specific chemical mediators such as nitric oxide, serotonin receptors, calcitonin gene-related peptide, and prostanoids have been found to play a role in the headache phase of a typical migraine attack. And in the postdromal phase, in which some migraine symptoms persist after the headache ends, is characterized by persistent blood flow changes in certain regions of the brain [20]. In our study, CAD was associated with an increased risk (HR 4.62) for BPPV in migraineurs. This finding could possibly echo the assumption that vasospasm and the downstream vascular events that take place intracranially as well as in the inner ear may at least partially explain the relationship between migraine and BPPV. Further investigations would be needed to clarify the underlying causes for the link between migraine and BPPV.

Our study has a number of clinical implications. Physicians must be more alert to vestibular complaints of migraine patients, especially in those older than 40 years, with CAD, or who seek frequent OPD help for migraine, since they are more likely to develop BPPV. Early recognition and prompt management of BPPV are important in resolving patients' discomforts. Finally, migraine history should be routinely inquired about in patients with BPPV. Examining migraine history can be used to clarify the etiology and to delineate whether BPPV development in migraineurs could have a different clinical pattern or prognosis.

The followings are limitations of this study. First, deviations in coding of the claims data were inevitable. A matched control cohort was used to offset the possible coding errors and to minimize bias. The Bureau of the NHI routinely and randomly samples a fixed percentage of claims from every contracted medical institution, and the accuracy of coding is audited by an independent group of physicians [13, 24, 25]. Second, it is difficult to explore the prognosis of BPPV, since the NHIR database is an administrative database that lacks detailed clinical data on the severity and outcome of BPPV. Third, the small sample size of certain subgroups might have contributed to failure in showing migraine as a significant risk factor in the subgroup analyses. Fourth, a major differential diagnosis is central positional vertigo due to a lesion in the cerebellum and its connection to the vestibular nuclei, and in addition, positional nystagmus can sometimes be seen during vestibular migraine attacks. However, in this study we tried to achieve high validity by obtaining coding from a neurologist or an otorhinolaryngologist. Therefore, we believe this considerably improved the quality of coding.

\section{Conclusions}

In conclusion, our study demonstrated that patients with migraine had a 2.03-fold greater risk of developing BPPV compared to the age- and sex-matched control cohort. Although BPPV is a rare condition, vestibular symptoms in migraine patients should still alert physicians to the possibility of occurrence of BPPV, especially those who are older than 40 , have $\mathrm{CAD}$, or who make frequent OPD visits for migraine.

\section{Abbreviations}

BPPV: Benign paroxysmal positional vertigo; CAD: Coronary artery disease; Cls: Confidence intervals; COPD: Chronic obstructive pulmonary disease; HR: Hazard ratio; ICD-9-CM: International Classification of Diseases, Ninth Revision, Clinical Modification; IRRs: Incidence rate ratios; NHI: National Health Insurance; NHRI: National Health Research Institute; OPD: Outpatient department; SSNHL: Sudden sensorineural hearing loss.

\section{Competing interests}

The authors declare that there is no conflict of interest.

\section{Authors' contributions}

$\mathrm{CH}$ participated in study design, interpreted result and drafted the manuscript. CJ participated in study design and performed the statistical analysis. LY participated in study design and helped to draft the manuscript. TJ helped to perform the statistical analysis and coordination. SJ conceived of the study, participated in its design, coordination and revising the manuscript. All authors read and approved the final manuscript.

\section{Acknowledgement}

This study was supported in part by grants from Taiwan National Science Council (100-2314-B-010-019-MY2, 100-2314-B-010-018-MY3), Taipei Veterans General Hospital (VGHUST101-G7-1-1, V101C-106, V101E7-003), NSC support for the Center for Dynamical Biomarkers and Translational Medicine, National Central University, Taiwan (NSC 100-2911-I-008-001), Brain Research Center, National Yang-Ming University and a grant from Ministry of Education, Aim for the Top University Plan. Miss Yeh, Chiu-Mei made a great contribution to the revision of this manuscript on the calculation and interpretation of statistical analyses.

\section{Author details}

'Department of Otorhinolaryngology-Head and Neck Surgery, Taipei Veterans General Hospital, Taipei, Taiwan. ${ }^{2}$ Division of Hematology and Oncology, Department of Medicine, Taipei Veterans General Hospital, Taipei, Taiwan. ${ }^{3}$ Division of Endocrinology and Metabolism, Department of Medicine, Taipei Veterans General Hospital, Taipei, Taiwan. ${ }^{4}$ Department of Family Medicine, Taipei Veterans General Hospital, Taipei, Taiwan. ${ }^{5}$ Department of Neurology, Neurological Institute, Taipei Veterans General Hospital, Taipei, Taiwan.

${ }^{6}$ Department of Otorhinolaryngology, National Yang-Ming University School of Medicine, Taipei, Taiwan. ${ }^{7}$ Institute of Public Health, National Yang-Ming University School of Medicine, Taipei, Taiwan. ${ }^{8}$ Department of Medicine, National Yang-Ming University School of Medicine, Taipei, Taiwan. ${ }^{9}$ Department of Neurology, National Yang-Ming University School of Medicine, Taipei, Taiwan. ${ }^{10}$ Institute of Brain Science, National Yang-Ming University School of Medicine, Taipei, Taiwan. ${ }^{11}$ Brain Research Center, National Yang-Ming University School of Medicine, Taipei, Taiwan.

Received: 22 April 2015 Accepted: 24 June 2015

Published online: 04 July 2015

\section{References}

1. Baloh RW (1997) Neurotology of migraine. Headache 37(10):615-621

2. Kayan A, Hood JD (1984) Neuro-otological manifestations of migraine. Brain 107(Pt 4):1123-1142 
3. von Brevern M, Neuhauser H (2011) Epidemiological evidence for a link between vertigo and migraine. J Vestib Res 21:299-304

4. Wang SJ, Fuh JL, Young YH, Lu SR, Shia BC (2000) Prevalence of migraine in Taipei, Taiwan: a population-based survey. Cephalalgia 20:566-572

5. Wang SJ, Chen PK, Fuh JL (2010) Comorbidities of migraine. Front Neurol 1:16

6. Ferrari MD (2013) Headache: the changing migraine brain. Lancet Neurol 12:6-8

7. Narbone MC, Leggiadro N, La Spina P, Rao R, Grugno R, Musolino R (1996) Migraine stroke: a possible complication of both migraine with and without aura. Headache 36:481-483

8. Kim JS, Zee DS (2014) Clinical practice. Benign paroxysmal positional vertigo. N Engl J Med 370:1138-1147

9. von Brevern M, Radtke A, Lezius F, Feldmann M, Ziese T, Lempert T, Neuhauser $\mathrm{H}$ (2007) Epidemiology of benign paroxysmal positional vertigo: a population based study. J Neurol Neurosurg Psychiatry 78:710-715

10. Parnes LS, Agrawal SK, Atlas J (2003) Diagnosis and management of benign paroxysmal positional vertigo (BPPV). CMAJ 169:681-693

11. Bisdorff A (2014) Migraine and dizziness. Curr Opin Neurol 27:105-110

12. Porta-Etessam J, Garcia-Cobos R, Cuadrado ML, Casanova I, Lapena T, Garcia-Ramos R (2011) Neuro-otological symptoms in patients with migraine. Neurologia 26(2):100-4

13. National Health Insurance Administration (2015) M.o.H., Taiwan, National Health Insurance in Taiwan 2014-2015, Annual Report (bilingual). National Health Insurance Administration, Ministry of Health, Executive Yuan, Taiwan, p 5

14. Phillips J, Longridge N, Mallinson A, Robinson G (2010) Migraine and vertigo: a marriage of convenience? Headache 50:1362-1365

15. Calhoun AH, Ford S, Pruitt AP, Fisher KG (2011) The point prevalence of dizziness or vertigo in migraine-and factors that influence presentation. Headache 51:1388-1392

16. Warninghoff JC, Bayer O, Ferrari U, Straube A (2009) Co-morbidities of vertiginous diseases. BMC Neurol 9:29

17. Uneri A (2004) Migraine and benign paroxysmal positional vertigo: an outcome study of 476 patients. Ear Nose Throat J 83:814-815

18. Faralli M, Cipriani L, Del Zompo MR, Panichi R, Calzolaro L, Ricci G (2014) Benign paroxysmal positional vertigo and migraine: analysis of 186 cases. B-ENT 10:133-139

19. Ishiyama A, Jacobson KM, Baloh RW (2000) Migraine and benign positional vertigo. Ann Otol Rhinol Laryngol 109:377-380

20. Charles A (2013) The evolution of a migraine attack - a review of recent evidence. Headache 53:413-419

21. Evans RW, Ishiyama G (2009) Migraine with transient unilateral hearing loss and tinnitus. Headache 49:756-758

22. Chu CH, Liu CJ, Fuh JL, Shiao AS, Chen TJ, Wang SJ (2013) Migraine is a risk factor for sudden sensorineural hearing loss: a nationwide population-based study. Cephalalgia 33:80-86

23. Vass Z, Steyger PS, Hordichok AJ, Trune DR, Jancso G, Nuttall AL (2001) Capsaicin stimulation of the cochlea and electric stimulation of the trigeminal ganglion mediate vascular permeability in cochlear and vertebrobasilar arteries: a potential cause of inner ear dysfunction in headache. Neuroscience 103(1):189-201

24. Cheng CL, Lee CH, Chen PS, Li YH, Lin SJ, Yang YH (2014) Validation of acute myocardial infarction cases in the national health insurance research database in taiwan. J Epidemiol 24:500-507

25. Cheng CL, Kao YH, Lin SJ, Lee CH, Lai ML (2011) Validation of the National Health Insurance Research Database with ischemic stroke cases in Taiwan. Pharmacoepidemiol Drug Saf 20:236-242

\section{Submit your manuscript to a SpringerOpen ${ }^{\odot}$ journal and benefit from:}

- Convenient online submission

$\checkmark$ Rigorous peer review

- Immediate publication on acceptance

- Open access: articles freely available online

- High visibility within the field

- Retaining the copyright to your article

Submit your next manuscript at $>$ springeropen.com 